\title{
Critical Frequencies of Composite Cylindrical Shells
}

\author{
K. Renji \\ Advanced Technology Development Group, ISRO Satellite center, ISRO Vimanapura Post, Bangalore, India \\ 560017.
}

\author{
S. Josephine Kelvina Florence \\ Structures Group, ISRO Satellite center, ISRO Vimanapura Post, Bangalore, India 560017.
}

(Received 10 October 2018; accepted 15 February 2019)

The sound radiation characteristics of a structure depend on its critical frequency. The expression for theoretically estimating the critical frequency of a composite cylindrical shell has not yet been reported. Thus, the practice is to use the expression for the composite panel for determining the critical frequency of a composite shell. In this work, critical frequencies of composite shells are investigated. As the critical frequency depends on the speed of the bending wave, an expression for the speed of the bending wave is first derived. It is seen that the curvature causes an increase in the speed of the bending wave and the orthotropic nature of the cylinder reduces the speed. An expression for the critical frequency of a composite cylindrical shell is then derived. The curvature causes a reduction in the critical frequency and the influence is significant in acoustically thick cylinders. Hence, the critical frequencies of such cylinders cannot be determined by using the expression for the panels. Effects of transverse shear deformation on the speed of the bending wave as well as the critical frequency are then investigated. Transverse shear deformation causes both reduction in the speed of the bending wave and an increase in the critical frequency. The orthotropic nature of the cylindrical shell increases the critical frequency further. The critical frequency of a typical composite cylinder is determined through a numerical simulation and the results are in agreement with the results obtained using the expressions derived. The critical frequency of a typical composite cylinder obtained through an experiment is presented. With this work, expressions for theoretically estimating the speeds of the bending waves and critical frequencies are derived for a composite cylindrical shell considering transverse shear deformation.

\section{NOMENCLATURE}

Symbols not listed here are used only at specific places and are explained wherever they occur.

$A_{i j} \quad$ extensional stiffness terms

$B_{i j} \quad$ coupling stiffness terms

$D_{i j} \quad$ bending stiffness terms

$D \quad$ Bending stiffness of the plate

$E_{f} \quad$ Young's modulus of the face-sheet

$F_{a} \quad$ actual force

$F_{m} \quad$ measured force

Img imaginary part

$L \quad$ length of the cylindrical shell

$M \quad$ Mass of the impedance head and attachment elements

$N \quad$ shear rigidity in $\mathrm{N} / \mathrm{m}$

Re real part

$Y_{M} \quad$ admittance of the impedance head and the attachment elements

$Y_{a} \quad$ actual admittance

$Y_{m} \quad$ measured admittance

a radius of the cylindrical shell

$c_{b, p} \quad$ wave speed of bending wave in plates

$c_{b} \quad$ wave speed of bending wave in shells

$f_{c} \quad$ critical frequency of cylinder in $\mathrm{Hz}$

$f_{r} \quad$ ring frequency of cylinder in $\mathrm{Hz}$

$k_{x}, k_{y}$ circumferential and axial wave numbers

$t_{c} \quad$ thickness of the core $t_{f} \quad$ thickness of face sheet

$\frac{m \pi}{L}, \frac{n}{a} \quad$ function of axial and circumferential wave number

$t \quad$ thickness of the isotropic cylinder/plate

$u_{x}, u_{\theta}$ in-plane displacements

$u_{r} \quad$ radial/transverse displacement

$\eta_{\text {total }}$ Total loss factor

$\mu \quad$ Poisson's ratio

$\rho_{m} \quad$ mass per unit area

$\rho_{v} \quad$ density of the material

$\omega_{c, p} \quad$ critical frequency of composite plate in $\mathrm{rad} / \mathrm{sec}$

$\omega_{c} \quad$ critical frequency of cylinder in $\mathrm{rad} / \mathrm{sec}$

$\omega_{r} \quad$ ring frequency of cylinder in $\mathrm{rad} / \mathrm{sec}$

\section{INTRODUCTION}

Sound radiation characteristics of cylindrical shells are characterized by the parameter critical frequency. ${ }^{1}$ If excited at the critical frequency, the speed of the free bending waves generated in the structure is equal to the speed of sound in air, the medium in which the sound is radiated. At the critical frequency the wavenumber of the bending wave in the structure is equal to the wavenumber in the air causing significant sound radiation. At frequencies below the critical frequency the wavenumber in the structure is larger than the wavenumber in the air, reducing the sound radiation. Therefore, it is essential to determine the critical frequencies of a structure when studying its sound radiation characteristics.

The expression for theoretically estimating the critical fre- 
quencies of isotropic as well as composite flat panels are reported. $^{2}$ Those works include the transverse shear deformation as well. Transverse shear deformation influences the critical frequencies of honeycomb sandwich panels. In the case of shells, it is known that the curvature of the cylinder causes an increase in the speed of the bending wave ${ }^{3}$ and therefore it is expected that the critical frequency of the cylindrical shells will be less than the critical frequencies estimated using an expression applicable for flat plates.

However, the critical frequencies of cylindrical shells are still determined using the expressions applicable for the flat plates, ${ }^{4-6}$ which is valid only under certain conditions. It is important to remember that the response characteristics of cylindrical shells to acoustic excitation are influenced by yet another parameter of interest, i.e. the ring frequency. Ring frequency is the frequency at which a cylinder undergoes uniform expansion and contraction. At frequencies greater than the ring frequency, the stiffening due to curvature reduces and it behaves like a plate. At frequencies well above the ring frequency, the behavior of a cylindrical shell can be approximated to the behavior of flat plates. Therefore, if the critical frequency is very much higher than the ring frequency, the critical frequency will be unaffected by the curvature and hence the expressions for the flat plates can be used to estimate the critical frequency of such cylindrical shells. However, if the critical frequency is lower than the ring frequency, it is expected that the critical frequency will be influenced by the curvature and the expressions for the flat plates will no longer hold well. Critical frequencies of such shells are not reported. Although Ghinet et al. ${ }^{6}$ presented an expression for determining the critical frequency of composite structures, they are applicable only for panels and not for shells.

The critical frequencies of composite cylindrical shells are investigated in the present work. To determine the critical frequency, the speed of the bending wave must be obtained first. The expression for the critical frequency of an isotropic cylinder, for which an expression for the bending wave is already available ${ }^{3}$ is derived first. Expressions for determining the speed of the free bending wave and then the critical frequency are then derived for composite cylindrical shells. As the speed of the bending wave depends on the transverse shear deformation, the transverse shear effects are then incorporated in these expressions. The influence of the various parameters of the critical frequency is discussed. The results for a typical composite cylinder are presented. The results using the derived expressions are then compared with the results of numerical simulations and experiments.

\section{ISOTROPIC CYLINDRICAL THIN SHELLS}

In isotropic shells, the speed of the bending wave of a mode order is higher than the speed of the corresponding mode in an equivalent plate. $^{3}$ Therefore, the critical frequency of the shell is expected to be lower than the critical frequency of the equivalent plate and the expression for the critical frequency is derived here. The equivalent plate has the same dimensions and cross section as the cylinder, the circumference of the cylinder being one dimension of the plate.

\subsection{Speed of Bending Waves}

Though the characteristics of free bending waves in isotropic shells are well known, for continuity they are briefed here. The expression for the natural frequency of a cylindrical shell was cast in terms of wavenumbers as

$$
\omega_{m n}^{2}=\frac{D}{\rho_{m}} k^{4}+\left(1-\mu^{2}\right) \frac{E}{\rho_{V}\left(1-\mu^{2}\right) a^{2}} \frac{k_{y}^{4}}{\left(k_{x}^{2}+k_{y}^{2}\right)^{2}} .
$$

The wavenumber in the structure was denoted by $k$ with circumferential wavenumber of $k_{x}$ and the longitudinal wavenumber of $k_{y}$. They were related by

$$
\omega_{m n}^{2}=\frac{D}{\rho_{m}} k^{4}+\left(1-\mu^{2}\right) \omega_{r}^{2} \frac{k_{y}^{4}}{k^{4}} .
$$

The first term in the above expression was the natural frequency of the equivalent plate for the same mode order, denoted by $\omega_{m n, p}$. The natural frequency of the shell was then written in terms of the natural frequency of the plate as

$$
\omega_{m n}^{2}=\omega_{m n, p}^{2}+\left(1-\mu^{2}\right) \omega_{r}^{2} \frac{k_{y}^{4}}{k^{4}}
$$

The effect of curvature was to increase the natural frequency. The influence was significant for low values of circumferential wavenumber and large values of longitudinal wavenumber.

The speed of the bending wave in the shell, denoted by $c_{b}$, was then determined from the natural frequency as

$$
c_{b}^{2}=\frac{c_{b, p}^{2}}{1-\frac{\left(1-\mu^{2}\right)}{\left(\omega / \omega_{r}\right)^{2}} \frac{k_{y}^{4}}{k^{4}}} ;
$$

where $c_{b, p}$ was the speed of the bending wave in the plate structure. It is to be noted that $k, \omega$ and $c_{b}$ corresponded with certain values of $m, n$. The speed of the bending wave was increased due to curvature. The results are identical to those given by Manning and Maidanik. ${ }^{3}$

\subsection{Critical Frequency}

Consider the expression for natural mode, given by Eq. (1). When $\omega_{m n}=\omega_{c}, c_{b}=c$ where $c$ is the speed of sound in air. Therefore at $\omega_{c}$

$$
\omega_{c}^{2}=\frac{D}{\rho_{m}}\left(\frac{\omega_{c}}{c}\right)^{4}+\left(1-\mu^{2}\right) \omega_{r}^{2} \frac{k_{y}^{4}}{k^{4}}
$$

As $\omega_{c, p}^{2}=\frac{c^{4} \rho_{m}}{D}$, Eq. (5) was written as

$$
\omega_{c}^{2}=\frac{\omega_{c}^{4}}{\omega_{c, p}^{2}}+\left(1-\mu^{2}\right) \omega_{r}^{2} \frac{k_{y}^{4}}{k^{4}}
$$

The above equation was then re-cast as

$$
\omega_{c}^{2}=\omega_{c, p}^{2}\left[1-\left(1-\mu^{2}\right)\left(\frac{\omega_{r}}{\omega_{c}}\right)^{2} \frac{k_{y}^{4}}{k^{4}}\right] .
$$

The critical frequency of the shell is less than that of the equivalent plate. The effect of curvature is to reduce the critical frequency.

It can also be noted that the influence of curvature is significant for higher values of $\omega_{r} / \omega_{c}$. If $\omega_{r} \ll \omega_{c}, \omega_{c} \approx \omega_{c, p}$ 
(for example if $\omega_{r} / \omega_{c}=0.5, \omega_{c}=0.99 \omega_{c, p}$ ). If $\omega_{r} \gg \omega_{c}$, $\omega_{c} \ll \omega_{c, p}$ (for example if $\omega_{r} / \omega_{c}=2.0, \omega_{c}=0.78 \omega_{c, p}$ ). This means that the influence of curvature becomes important when the ring frequency is higher than the critical frequency. If the critical frequency is higher than the ring frequency, the curvature does not influence the value of the critical frequency and the critical frequency can be determined using the expression for the plate. If the critical frequency is lower than the ring critical frequency, the curvature influences the critical frequency and the critical frequency of the shell is less than the critical frequency of the equivalent plate. In such cases the critical frequency cannot be estimated using the expression for the plate and the expression derived here is required to determine the critical frequency.

\section{COMPOSITE SHELLS}

In isotropic shells, the speed of the bending wave of a mode order is higher than the speed of the corresponding mode in an equivalent plate. ${ }^{6}$ In the same manner, the speeds of the free bending waves in composite cylinders are expected to be higher than those of equivalent plates. The speeds of the free bending waves in composite plates are well known, including the effects of transverse shear deformation and orthotropic nature of the plates. ${ }^{2}$ However, the speed of the bending waves in a composite cylinder and its critical frequency are not reported and this is done here.

To proceed, it was assumed that the shell was cylindrical and shallow; the term $\left[1+\frac{z}{a}\right]$ was very close to one, i.e., the radius of curvature is very large. The laminate considered was symmetric $\left(B_{i j}=0\right)$, balanced $\left(A_{16}, A_{26}=0\right)$ and orthotropic $\left(D_{16}, D_{26}=0\right)$. The plane stress condition existed and $\epsilon_{r}=0$, i.e., the displacement $u_{r}$ was independent of $z$. The mass distribution was uniform and rotary inertia was neglected. These assumptions were generally applied to all such laminate formulations. Additionally, the displacements $u_{x}$ and $u_{\theta}$ were related by Airy's stress function and not independent. The influence of inertia force in the in-plane direction was neglected.

\subsection{Speed of Bending Waves}

The expression for the natural frequency of a thin composite shell is given by ${ }^{7,8}$

$$
\begin{aligned}
& \omega_{m n}^{2}=\frac{1}{\rho_{m}}\left\{D_{11}\left(\frac{m \pi}{L}\right)^{4}+2\left(D_{12}+2 D_{66}\right)\left(\frac{m \pi}{L}\right)^{2}\left(\frac{n}{a}\right)^{2}+\right. \\
& D_{22}\left(\frac{n}{a}\right)^{4}+ \\
& \left.\frac{\left(\frac{m \pi}{L}\right)^{4} \frac{A_{11} A_{22}-A_{12}^{2}}{a^{2}}}{A_{11}\left(\frac{m \pi}{L}\right)^{4}+A_{22}\left(\frac{n}{a}\right)^{4}+\frac{A_{11} A_{22}-A_{12}^{2}-2 A_{12} A_{66}}{A_{66}}\left(\frac{m \pi}{L}\right)^{2}\left(\frac{n}{a}\right)^{2}}\right\} ;
\end{aligned}
$$

with $A_{i j}$ and $D_{i j}$ are as per conventional definition for composite laminates. ${ }^{9}$ This was then re-cast in terms of wavenumbers as

$$
\begin{gathered}
\omega_{m n}^{2}=\frac{1}{\rho_{m}}\left\{D_{11} k_{y}^{4}+2\left(D_{12}+2 D_{66}\right) k_{x}^{2} k_{y}^{2}+D_{22} k_{x}^{4}+\right. \\
\left.\frac{\frac{A_{11} A_{22}-A_{12}^{2}}{a^{2}} k_{y}^{4}}{A_{11} k_{y}^{4}+A_{22} k_{x}^{4}+\frac{A_{11} A_{22}-A_{12}^{2}-2 A_{12} A_{66}}{A_{66}} k_{x}^{2} k_{y}^{2}}\right\}
\end{gathered}
$$

In Eq. (9), the first term represented the natural frequency of the plate and the second term signified the influence of the curvature. The natural frequency of the shell was then written in terms of the natural frequency of the equivalent plate as

$$
\begin{aligned}
& \omega_{m n}^{2}=\omega_{m n, p}^{2}+ \\
& \frac{1}{\rho_{m}} \frac{\frac{A_{11} A_{22}-A_{12}^{2}}{a^{2}} k_{y}^{4}}{A_{11} k_{y}^{4}+A_{22} k_{x}^{4}+\frac{A_{11} A_{22}-A_{12}^{2}-2 A_{12} A_{66}}{A_{66}} k_{x}^{2} k_{y}^{2}} .
\end{aligned}
$$

Denoting $\delta=A_{22} / A_{11}, \alpha=A_{12} / A_{11}$ and $\beta=A_{66} / A_{11}$, the second term became

$$
2^{\text {nd }} \text { term }=\frac{1}{\rho_{m} a^{2}} \frac{\left(A_{22}-\alpha A_{12}\right) k_{y}^{4}}{k_{y}^{4}+\delta k_{x}^{4}+\frac{\delta-\alpha^{2}-2 \alpha \beta}{\beta} k_{x}^{2} k_{y}^{2}} .
$$

For an orthotropic structure having the same properties in both the directions, i.e, $\delta=1$, the second term in the expression for the natural frequency became

$$
2^{\text {nd }} \text { term }=\frac{1}{\rho_{m} a^{2}} \frac{\left(A_{22}-\alpha A_{12}\right) k_{y}^{4}}{k_{y}^{4}+k_{x}^{4}+\frac{1-\alpha^{2}-2 \alpha \beta}{\beta} k_{x}^{2} k_{y}^{2}} .
$$

For large values of wavenumbers, it was further simplified as

$$
2^{\text {nd }} \text { term }=\frac{1}{\rho_{m} a^{2}} \frac{\left(A_{22}-\alpha A_{12}\right) k_{y}^{4}}{k^{4}} .
$$

The speed of the bending wave in the shell was determined from the natural frequency as

$$
c_{b}^{2}=c_{b, p}^{2}+\frac{2^{n d} \text { term }}{k^{2}} .
$$

Upon substituting the expression for the second term, i.e., Eq. (13) and introducing ring frequency, which is the frequency at which the shell undergoes uniform expansion and compression, given by

$$
\omega_{r}^{2}=\frac{A_{22}-\alpha A_{12}}{\rho_{m} a^{2}} .
$$

The expression for the speed of the bending wave in the shell considering Eq. (15), became

$$
c_{b}^{2}=c_{b, p}^{2}+\frac{\omega_{r}^{2}}{k^{2}}\left(\frac{k_{y}^{4}}{k^{4}}\right) .
$$

The speed of the bending wave had increased due to curvature. The above equation was re-cast in another convenient form as

$$
c_{b}^{2}=\frac{c_{b, p}^{2}}{1-\left(\omega_{r} / \omega\right)^{2}\left(\frac{k_{y}^{4}}{k^{4}}\right)}
$$

Equations (16) or (17) can be used for determining the speeds of the bending waves in composite shells. In deriving these relations we considered that the laminate was with $A_{11}=A_{22}$ and the wavenumbers were quite high, such that, $k_{x}^{2}$ and $k_{y}{ }^{2}$ are negligible compared to $k^{4}$. 


\subsection{Critical Frequency}

Consider the expression for natural frequency of a thin composite shell given by Eq. (9). For a structure with $D_{11}=$ $D_{22}=D$, at critical frequency

$$
\begin{aligned}
& \omega_{c}^{2}=\frac{D}{\rho_{m}} \frac{\omega_{c}^{4}}{c^{4}}+\frac{1}{\rho_{m}} \\
& \left\{\frac{k_{y}^{4} \frac{A_{11} A_{22}-A_{12}^{2}}{a^{2}}}{A_{11} k_{y}^{4}+A_{22} k_{x}^{4}+\frac{A_{11} A_{22}-A_{12}^{2}-2 A_{12} A_{66}}{A_{66}} k_{x}^{2} k_{y}^{2}}\right\} .
\end{aligned}
$$

Introducing the critical frequency of the equivalent plate into the above expression, the critical frequency of the shell becomes

$$
\begin{aligned}
& \omega_{c}^{2}=\frac{\omega_{c}^{4}}{\omega_{c, p}^{2}}+\frac{1}{\rho_{m}} \\
& \left\{\frac{k_{y}^{4} \frac{A_{11} A_{22}-A_{12}^{2}}{a^{2}}}{A_{11} k_{y}^{4}+A_{22} k_{x}^{4}+\frac{A_{11} A_{22}-A_{12}^{2}-2 A_{12} A_{66}}{A_{66}} k_{x}^{2} k_{y}^{2}}\right\} .
\end{aligned}
$$

Using Eq. (13) for the second term we get

$$
\omega_{c}^{2}=\frac{\omega_{c}^{4}}{\omega_{c, p}^{2}}+\frac{1}{\rho_{m} a^{2}} \frac{\left(A_{22}-\alpha A_{12}\right) k_{y}^{4}}{k^{4}} .
$$

Introducing ring frequency into the above expression,

$$
\omega_{c}^{2}=\frac{\omega_{c}^{4}}{\omega_{c, p}^{2}}+\left(\omega_{r}\right)^{2} \frac{k_{y}^{4}}{k^{4}}
$$

Re-arranging the terms

$$
\omega_{c, p}^{2}=\omega_{c}^{2}+\left(\frac{\omega_{r}}{\omega_{c}}\right)^{2} \frac{k_{y}^{4}}{k^{4}} \omega_{c, p}^{2}
$$

Therefore, the critical frequency of the shell was

$$
\omega_{c}^{2}=\omega_{c, p}^{2}\left[1-\left(\frac{\omega_{r}}{\omega_{c}}\right)^{2} \frac{k_{y}^{4}}{k^{4}}\right] .
$$

Equation (23) was used to determine the critical frequency of a composite thin shell. The characteristics were identical to those of isotropic shells, the curvature influenced the critical frequency if the critical frequency was lower than the ring frequency, otherwise the influence was negligible.

\section{INFLUENCE OF TRANSVERSE SHEAR}

The speeds of the free bending waves in plates were influenced by the transverse shear deformation, ${ }^{2}$ especially at higher frequencies. Similar characteristics were expected for the bending waves in shells too and therefore influenced the critical frequency. This is investigated here.

Here, the transverse deflection was considered to be due to both shear force and the bending moment. Mindlin's theory was used to represent the transverse shear deformation as per which the transverse plane remained straight but not normal to the mid surface after deformation. The rotation of the transverse plane $\left(\gamma_{x r}\right.$ and $\left.\gamma_{\theta r}\right)$ was constant along the thickness of the shell. But the transverse shear strains in the face sheets were neglected.

\subsection{Speed of Bending Waves}

The expression for the natural frequency of a thick composite shell was given in Eq. (24) (see top of the page). ${ }^{10}$

This was written in terms of wavenumbers as

$$
\begin{aligned}
& \left\{D_{11} k_{y}^{4}+2\left(D_{12}+2 D_{66}\right) k_{x}^{2} k_{y}^{2}+D_{22} k_{x}^{4}+\right. \\
& \left.\frac{\frac{A_{11} A_{22}-A_{12}^{2}}{a} k_{y}^{2} \times\left\{\frac{1}{a} k_{y}^{2}+\frac{1}{a N}\left(D_{11} k_{y}^{4}+D_{22} k_{x}^{2} k_{y}^{2}\right)\right\}}{A_{11} k_{y}^{4}+A_{22} k_{x}^{2}+\frac{A_{11} A_{22}-A_{12}^{2}-2 A_{12} A_{66}}{A_{66}} k_{x}^{2} k_{y}^{2}}\right\} .
\end{aligned}
$$

In Eq. (25), the first term represented the natural frequency of the equivalent plate and the second term signified the influence of the curvature. The natural frequency of the shell was then written in terms of the natural frequency of the plate as

$$
\begin{aligned}
& \omega_{m n}^{2}=\omega_{m n, p}^{2}+\frac{1}{\rho_{m}\left[1+\left(\frac{D_{11}}{N} k_{y}^{2}+\frac{D_{22}}{N} k_{x}^{2}\right)\right]} \times \\
& \frac{\frac{A_{11} A_{22}-A_{12}^{2}}{a} k_{y}^{2} \times\left\{\frac{1}{a} k_{y}^{2}+\frac{1}{a N}\left(D_{11} k_{y}^{4}+D_{22} k_{x}^{2} k_{y}^{2}\right)\right\}}{A_{11} k_{y}^{4}+A_{22} k_{x}^{2}+\frac{A_{11} A_{22}-A_{12}^{2}-2 A_{12} A_{66}}{A_{66}} k_{x}^{2} k_{y}^{2}} .
\end{aligned}
$$

Using the parameters $\alpha, \beta$ and $\delta$ and when $D_{11}=D_{22}=D$, the second term became

$$
2^{\text {nd }} \text { term }=\frac{1}{\rho_{m} a^{2}\left[1+\frac{D}{N} k^{2}\right]} \times \frac{\left(A_{22}-\alpha A_{12}\right) k_{y}^{4}\left[1+\frac{D}{N} k^{2}\right]}{k_{y}^{4}+\delta k_{x}^{4}+\frac{\delta-\alpha^{2}-2 \alpha \beta}{\beta} k_{x}^{2} k_{y}^{2}} .
$$

For an orthotropic structure having the same properties in both the directions, i.e, $\delta=1$, the second term in the expression for the natural frequency became

$$
2^{\text {nd }} \text { term }=\frac{1}{\rho_{m} a^{2}} \times \frac{\left(A_{22}-\alpha A_{12}\right) k_{y}^{4}}{k_{y}^{4}+k_{x}^{4}+\frac{1-\alpha^{2}-2 \alpha \beta}{\beta} k_{x}^{2} k_{y}^{2}} .
$$

For large values of wavenumbers, it was simplified further as

$$
2^{\text {nd }} \text { term }=\frac{\left(A_{22}-\alpha A_{12}\right)}{\rho_{m} a^{2}} \times \frac{k_{y}^{4}}{k^{4}} .
$$

The speed of the bending wave in the shell was determined from the natural frequency as

$$
c_{b}^{2}=c_{b, p}^{2}+\frac{2^{n d} \text { term }}{k^{2}} .
$$

Substituting for the expression for the second term, i.e., Eq. (29) and with the ring frequency given by Eq. (15), the speed of the bending wave in the shell was given by

$$
c_{b}^{2}=c_{b, p}^{2}+\frac{\omega_{r}^{2}}{k^{2}} \frac{k_{y}^{4}}{k^{4}} .
$$

The speed of the bending wave increased due to curvature. The above equation was re-cast in another convenient form as

$$
c_{b}^{2}=\frac{c_{b, p}^{2}}{1-\left(\omega_{r} / \omega\right)^{2}\left(\frac{k_{y}^{4}}{k^{4}}\right)} .
$$

Equations (31) or (32) can be used for determining the speeds of the bending waves in composite shells incorporating transverse shear deformation. 


$$
\begin{aligned}
& \omega_{m n}^{2}=\frac{1}{\rho_{m}\left[1+\left(\frac{D_{11}}{N}\left(\frac{m \pi}{L}\right)^{2}+\frac{D_{22}}{N}\left(\frac{n}{a}\right)^{2}\right)\right]}\left\{D_{11}\left(\frac{m \pi}{L}\right)^{4}+2\left(D_{12}+2 D_{66}\right)\left(\frac{m \pi}{L}\right)^{2}\left(\frac{n}{a}\right)^{2}+D_{22}\left(\frac{n}{a}\right)^{4}+\right. \\
& \left.\frac{\frac{A_{11} A_{22}-A_{12}^{2}}{a}\left(\frac{m \pi}{L}\right)^{2} \times\left\{\frac{1}{a}\left(\frac{m \pi}{L}\right)^{2}+\frac{1}{a N}\left(D_{11}\left(\frac{m \pi}{L}\right)^{4}+D_{22}\left(\frac{m \pi}{L}\right)^{2}\left(\frac{n}{a}\right)^{2}\right)\right\}}{A_{11}\left(\frac{m \pi}{L}\right)^{4}+A_{22}\left(\frac{n}{a}\right)^{4}+\frac{A_{11} A_{22}-A_{12}^{2}-2 A_{12} A_{66}}{A_{66}}\left(\frac{m \pi}{L}\right)^{2}\left(\frac{n}{a}\right)^{2}}\right\} .
\end{aligned}
$$

\subsection{Critical Frequency}

The natural frequency of a thick composite shell was given by (assume $D_{11}=D_{22}=D$ )

$$
\begin{aligned}
& \omega_{m n}^{2}=\frac{D}{\rho_{m}\left[1+\frac{D}{N} k^{2}\right]} \\
& \left\{k_{y}^{4}+k_{x}^{4}+\frac{2\left(D_{12}+2 D_{66}\right)}{D} k_{x}^{2} k_{y}^{2}+\right. \\
& \left.\frac{1}{D} \frac{\frac{A_{11} A_{22}-A_{12}^{2}}{a^{2}} k_{y}^{4}\left[1+\frac{D}{N} k^{2}\right]}{A_{11} k_{y}^{4}+A_{22} k_{x}^{2}+\frac{A_{11} A_{22}-A_{12}^{2}-2 A_{12} A_{66}}{A_{66}} k_{x}^{2} k_{y}^{2}}\right\} .
\end{aligned}
$$

At critical frequency

$$
\begin{aligned}
& \omega_{c}^{2}=\frac{D}{\rho_{m}\left[1+\frac{D}{N} k^{2}\right]} \frac{\omega_{c}^{4}}{c^{4}}+ \\
& \frac{1}{\rho_{m} a^{2}\left[1+\frac{D}{N} k^{2}\right]} \\
& \frac{\left(A_{11} A_{22}-A_{12}^{2}\right) k_{y}^{4}\left[1+\frac{D}{N} k^{2}\right]}{A_{11} k_{y}^{4}+A_{22} k_{x}^{4}+\frac{A_{11} A_{22}-A_{12}^{2}-2 A_{12} A_{66}}{A_{66}} k_{x}^{2} k_{y}^{2}} .
\end{aligned}
$$

Introducing the critical frequency of the equivalent plate, the critical frequency of the shell became

$$
\begin{aligned}
& \omega_{c}^{2}=\frac{\omega_{c}^{4}}{\omega_{c, p}^{2}}+\frac{1}{\rho_{m} a^{2}} \\
& \frac{\left(A_{11} A_{22}-A_{12}^{2}\right) k_{y}^{4}}{A_{11} k_{y}^{4}+A_{22} k_{x}^{4}+\frac{A_{11} A_{22}-A_{12}^{2}-2 A_{12} A_{66}}{A_{66}} k_{x}^{2} k_{y}^{2}} .
\end{aligned}
$$

Using Eq. (29) for the second term we got

$$
\omega_{c}^{2}=\frac{\omega_{c}^{4}}{\omega_{c, p}^{2}}+\frac{1}{\rho_{m} a^{2}} \frac{\left(A_{22}-\alpha A_{12}\right) k_{y}^{4}}{k^{4}} .
$$

Introducing the ring frequency, given by Eq. (15) into the above expression

$$
\omega_{c}^{2}=\frac{\omega_{c}^{4}}{\omega_{c, p}^{2}}+\left(\omega_{r}\right)^{2} \frac{k_{y}^{4}}{k^{4}} .
$$

Re-arranging the terms

$$
\omega_{c, p}^{2}=\omega_{c}^{2}+\left(\frac{\omega_{r}}{\omega_{c}}\right)^{2} \frac{k_{y}^{4}}{k^{4}} \omega_{c, p}^{2} .
$$

Therefore, the critical frequency of the shell was

$$
\omega_{c}^{2}=\omega_{c, p}^{2}\left[1-\left(\frac{\omega_{r}}{\omega_{c}}\right)^{2}\left(\frac{k_{y}}{k}\right)^{4}\right] .
$$

Eq. (39) was used to determine the critical frequency of a composite thick shell. The characteristics were identical to those of isotropic or composite thin shells, the curvature affecting the critical frequency. If the critical frequency is lower than the ring frequency, otherwise the influence is negligible.

It is to be noted that the expressions relating the critical frequency of the shell with the critical frequency of the plate were cast in identical forms, whether it was an isotropic thin shell, a composite thin shell, or composite thick shell. Depending on the type of shell, the critical frequency of the equivalent plate changed. In other words, the features of the type of crosssection and transverse shear effects were embedded in the critical frequency of the plate itself.

\section{CRITICAL FREQUENCIES OF A TYPICAL COMPOSITE CYLINDRICAL SHELL}

The critical frequency of a typical honeycomb sandwich composite cylinder was determined. The expressions derived in this work were employed in determining the critical frequency of a cylindrical shell with a honeycomb sandwich construction, provided the wavelength is far greater than the cell size. At lower frequencies both the face sheets vibrate together, and the model used for deriving the expression was valid. At higher frequencies, the core cells started vibrating, which lead to independent motions of the face sheets and hence the present formulation was not valid. In the case of the cylinder considered, the frequency at which the core started vibrating was estimated to be about $5560 \mathrm{~Hz}$, which was much higher than the critical frequency and hence the present expression was used.

The structural details of the cylinder considered are:

Length of the cylinder: $1.485 \mathrm{~m}$

Radius of the cylinder: $0.597 \mathrm{~m}$

Area of the cylinder: $\quad 5.578 \mathrm{~m}^{2}$

Face sheet thickness: $\quad 0.290 \mathrm{~mm}$

Face sheet material: 4 layers of CFRP:

$(0 / 90) / 35^{\circ} / 0^{\circ} /-35^{\circ}$

Core material: $\quad$ Aluminium honeycomb

Core height: $\quad 12 \mathrm{~mm}$

Core density: $\quad 32 \mathrm{~kg} / \mathrm{m}^{3}$

Core shear modulus: $\quad 1.4 \times 10^{8} \mathrm{~N} / \mathrm{m}^{2}$

Shear rigidity $\mathrm{N}$ : $\quad 17.6 \times 10^{5} \mathrm{~N} / \mathrm{m}$

The cross-section of the cylinder has the following elastic properties: $A_{11}=8.22 \times 10^{7} \mathrm{~N} / \mathrm{m}, A_{22}=3.3 \times 10^{7} \mathrm{~N} / \mathrm{m}$, $A_{12}=1.39 \times 10^{7} \mathrm{~N} / \mathrm{m}, A_{66}=1.48 \times 10^{7} \mathrm{~N} / \mathrm{m}, D_{11}=$ $3.11 \times 10^{3} \mathrm{Nm}, D_{22}=1.27 \times 10^{3} \mathrm{Nm}, D_{12}=5.20 \times 10^{2} \mathrm{Nm}$ and $D_{66}=5.55 \times 10^{2} \mathrm{Nm}$. Mass per unit area of the typical spacecraft cylinder was $1.72 \mathrm{~kg} / \mathrm{m}^{2}$.

The ring frequency of the cylinder was estimated as $1125 \mathrm{~Hz}$. As $D_{11} \neq D_{22}$, geometric mean of $D_{11}$ and $D_{22}$ is used as $D$. The critical frequencies were estimated with 


\begin{tabular}{|c|c|c|}
\hline \multirow[t]{2}{*}{ Type of cross-section } & \multicolumn{2}{|c|}{ Critical frequency } \\
\hline & Cylindrical shell & Equivalent Plate \\
\hline Thin isotropic & $390 \mathrm{~Hz}$ & $550 \mathrm{~Hz}$ \\
\hline Thin composite & $415 \mathrm{~Hz}$ & $565 \mathrm{~Hz}$ \\
\hline Isotropic with transverse shear & $470 \mathrm{~Hz}$ & $585 \mathrm{~Hz}$ \\
\hline Composite with transverse shear & $495 \mathrm{~Hz}$ & $600 \mathrm{~Hz}$ \\
\hline
\end{tabular}

$k_{y} / k=0.5$ and the results are given in Table 1.

Table 1 provides a comparison of critical frequencies of shells with those determined by idealising it as a plate, which is the existing practice. It also gives the influence of transverse shear flexibility as well as the orthotropic nature of the cross section on the critical frequency.

1. Curvature increases the speed of the bending wave. Therefore, the critical frequency of a cylindrical shell is less than that determined using the expression for plates. This result signifies the need of the present work.

2. The transverse shear deformation makes it more flexible in bending and hence the speed of the bending wave decreases if the transverse shear deformation is present. Therefore, the critical frequency increases due to transverse shear deformation.

3. The orthotropic nature makes the panel flexible compared to an isotropic nature. Therefore, it reduces speed of the bending wave and consequently the critical frequency increases.

The results given in Table 1 agree with these characteristics.

The critical frequency of the cylinder is estimated as $495 \mathrm{~Hz}$. If the curvature is neglected and the critical frequency is determined assuming it as a plate, the critical frequency would have been estimated as $600 \mathrm{~Hz}$, which is the existing practice. If the expression for the critical frequency of the cylinder is not derived considering the transverse shear deformation and the orthotropic nature, it would have been estimated as $390 \mathrm{~Hz}$ using the expression for thin cylinder. The above results show the need for the expressions derived in this work.

\section{RESULTS OF NUMERICAL SIMULATION}

The critical frequency of a cylindrical shell can be theoretically determined using the expressions derived in this work. Critical frequency can also be determined using a numerical simulation by finding the speed of the bending wave at each frequency and the frequency at which the speed of the bending wave is equal to the speed of sound in air will be the critical frequency. Though the expressions are derived from basic principles, a numerical simulation will help in validating the expressions derived. Hence, the critical frequency of a typical composite cylinder is obtained through a numerical simulation and compared with the results obtained using the expressions derived.

In a numerical simulation the critical frequency is obtained for a cylinder. This must be done in an iterative manner. Whereas using the expression derived in this work, the critical frequency can be directly estimated through an expression from the known parameters of the cylinder. The expression helps in understanding the parameters on which the critical frequency depends and its characteristics.
Numerical simulation is carried out in the following manner. The speed of the bending wave at a specific frequency can be determined from the wavenumber. At a given frequency, the wavenumber depends on the direction of the wave propagation and hence it is not unique. Therefore, at a given frequency there is no specific value for the wavenumber but it has a range of values. Therefore, the average value of the wavenumbers is determined using a numerical integration in the range of values of $\theta$ which is $0-\frac{\pi}{2}$. The expression for the natural frequency applicable for the shell is considered, which contains the terms involving axial and circumferential wave numbers. Following this, it is cast in a polar coordinate system. Once the wavenumber is found out, the flexural wave speed and critical frequency are determined.

\subsection{Composite Thin Shells}

Consider the natural frequency of a thin composite shell ${ }^{8}$ given by Eq. (8). For convenience it is reproduced here as

$$
\begin{aligned}
& \omega_{m n}^{2}=\frac{1}{\rho_{m}}\left\{D_{11}\left(\frac{m \pi}{L}\right)^{4}+2\left(D_{12}+2 D_{66}\right)\left(\frac{m \pi}{L}\right)^{2}\left(\frac{n}{a}\right)^{2}+\right. \\
& D_{22}\left(\frac{n}{a}\right)^{4}+ \\
& \left.\frac{\left(\frac{m \pi}{L}\right)^{4} \frac{A_{11} A_{22}-A_{12}^{2}}{a^{2}}}{A_{11}\left(\frac{m \pi}{L}\right)^{4}+A_{22}\left(\frac{n}{a}\right)^{4}+\frac{A_{11} A_{22}-A_{12}^{2}-2 A_{12} A_{66}}{A_{66}}\left(\frac{m \pi}{L}\right)^{2}\left(\frac{n}{a}\right)^{2}}\right\} . \\
& \text { Let } \frac{m \pi}{L}=k \cos \theta \& \frac{n}{a}=k \sin \theta \\
& \omega_{m n}^{2}=\frac{1}{\rho_{m}}\left\{k^{4}\left\{D_{11} c^{4}+2\left(D_{12}+2 D_{66}\right) c^{2} s^{2}+D_{22} s^{4}\right\}+\right. \\
& \left.\frac{c^{4}\left(A_{11} A_{22}-A_{12}^{2}\right)}{a^{2}\left(A_{11} c^{4}+A_{22} s^{4}+\frac{A_{11} A_{22}-A_{12}^{2}-2 A_{12} A_{66}}{A_{66}} c^{2} s^{2}\right)}\right\} . \quad
\end{aligned}
$$

Expression for the wave number is found out from Eq. (40) as

$k^{2}=\left\{\frac{\rho_{m} \omega_{m n}^{2}-\frac{c^{4}\left(A_{11} A_{22}-A_{12}^{2}\right)}{a^{2}\left(A_{11} c^{4}+A_{22} s^{4}+\frac{A_{11} A_{22}-A_{12}^{2}-2 A_{12} A_{66}}{A_{66}} c^{2} s^{2}\right)}}{D_{11} c^{4}+2\left(D_{12}+2 D_{66}\right) c^{2} s^{2}+D_{22} s^{4}}\right\}_{41)}^{\frac{1}{2}}$.

As discussed before, since the wavenumber at a specific frequency depends on the direction of propagation, the average value of the wavenumbers is determined numerically in the following way

$$
\begin{aligned}
& k=\frac{2}{\pi} \int_{0}^{\frac{\pi}{2}} \\
& \left\{\frac{\rho_{m} \omega_{m n}^{2}-\frac{c^{4}\left(A_{11} A_{22}-A_{12}^{2}\right)}{a^{2}\left(A_{11} c^{4}+A_{22} s^{4}+\frac{A_{11} A_{22}-A_{12}^{2}-2 A_{12} A_{66}}{\left.A_{66} c^{2}\right)}\right.}}{D_{11} c^{4}+2\left(D_{12}+2 D_{66}\right) c^{2} s^{2}+D_{22} s^{4}}\right\}^{\frac{1}{4}} d \theta .
\end{aligned}
$$

The speed of the wave can then be found out using the relation $c=\frac{\omega}{k}$. For an equivalent thin flat plate, wavenumber is found by setting radius of the shell to $\infty$.

$k_{\text {plate }}=\frac{2}{\pi} \int_{0}^{\frac{\pi}{2}}\left\{\frac{\rho_{m} \omega_{m n}^{2}}{D_{11} c^{4}+2\left(D_{12}+2 D_{66}\right) c^{2} s^{2}+D_{22} s^{4}}\right\}^{\frac{1}{4}} d \theta$.

Results thus obtained are presented in Table 2. 
Table 2. Speeds of the bending waves in thin composite shells and equivalent flat plates.

\begin{tabular}{|c|c|c|c|c||}
\hline S.No. & Frequency & \multicolumn{2}{|c|}{ Wave speed (m/s) } & Ratio w.r.t plate \\
\cline { 3 - 4 } & $(\mathrm{Hz})$ & Shell & Plate & \\
\hline 1 & 315 & 257 & 257 & 1.00 \\
\hline 2 & 400 & 315 & 290 & 1.09 \\
\hline 3 & 500 & 375 & 324 & 1.16 \\
\hline 4 & 630 & 444 & 364 & 1.22 \\
\hline 5 & 800 & 518 & 410 & 1.26 \\
\hline 6 & 1000 & 578 & 458 & 1.26 \\
\hline 7 & 1250 & 573 & 512 & 1.12 \\
\hline 8 & 1600 & 613 & 579 & 1.06 \\
\hline 9 & 2000 & 670 & 648 & 1.03 \\
\hline 10 & 2500 & 739 & 724 & 1.02 \\
\hline 11 & 3150 & 823 & 813 & 1.01 \\
\hline 12 & 4000 & 923 & 916 & 1.01 \\
\hline
\end{tabular}

Based on the results presented in Table 2, the critical frequency of the shell is expected to be about $445 \mathrm{~Hz}$, taking $343 \mathrm{~m} / \mathrm{s}$ as the speed of sound in air. It is $560 \mathrm{~Hz}$ if calculated neglecting the curvature. The results show the influence of the curvature and the need for the expression derived. The critical frequencies estimated using the expressions derived in this work are $415 \mathrm{~Hz}$ and $565 \mathrm{~Hz}$ respectively for the cylindrical shell and plate approximation. Results obtained through numerical integration validate the expressions derived and the important conclusions drawn in the previous chapter.

It was shown in previous chapters that the influence of the curvature on the critical frequency depends on its relative position with reference to the ring frequency. If the critical frequency is higher than the ring frequency, the curvature does not influence the critical frequency. However, the opposite happens if it is below the ring frequency. Therefore, it is important to determine the ring frequency. Below the ring frequency the speed of the bending wave is higher than the speed of the bending wave in the equivalent plate. Results obtained through the numerical simulation show that, at $1000 \& 1250 \mathrm{~Hz}$ frequency band, the speed of the bending wave in the cylinder is much higher than that in the equivalent plate. Beyond this frequency band, i.e., beyond $1250 \mathrm{~Hz}$, the behavior is identical to that of flat plates. Therefore, the ring frequency of this cylinder is between $1000 \mathrm{~Hz}$ and $1250 \mathrm{~Hz}$. The critical frequency of this cylinder is much lower than the ring frequency and thus it is an acoustically thick cylinder that results in significant influence of curvature on the critical frequency.

\subsection{Composite Thick Shells}

Natural frequency of a thick orthotropic cylinder ${ }^{10}$ given by Eq. (24), for convenience is reproduced at the top of the next page as $\left(^{*}\right)$. Defining the wave numbers as $\frac{m \pi}{L}=k \cos \theta \&$ $\frac{n}{a}=k \sin \theta$ and using a polar coordinate system in the wave space, the expression for $k^{2}$ can be obtained as

$$
k^{4} \frac{f_{1}}{\rho_{m} \omega_{m n}^{2}-f_{3}}-\frac{k^{2} f_{2}}{N}-1=0
$$

where $f_{1}, f_{2}$ and $f_{3}$ are functions of $\theta$ (given below) representing the orthotropic elastic properties $f_{1}=D_{11} c^{4}+$ $2\left(D_{12}+2 D_{66}\right) c^{2} s^{2}+D_{22} s^{4} ; f_{2}=D_{11} c^{2}+D_{22} s^{2} ; f_{3}=$
Table 3. Speeds of the bending waves in thick composite shells and equivalent flat plates.

\begin{tabular}{|c|c|c|c|c||}
\hline \multirow{2}{*}{ S.No } & Frequency $(\mathrm{Hz})$ & \multicolumn{2}{|c|}{ Wave speed $(\mathrm{m} / \mathrm{s})$} & \multirow{2}{*}{ Ratio w.r.t plate } \\
\cline { 3 - 4 } & & Shell & Plate & \\
\hline 1 & 315 & 266 & 253 & 1.05 \\
\hline 2 & 400 & 323 & 283 & 1.14 \\
\hline 3 & 500 & 382 & 315 & 1.21 \\
\hline 4 & 630 & 446 & 351 & 1.27 \\
\hline 5 & 800 & 511 & 392 & 1.30 \\
\hline 6 & 1000 & 558 & 433 & 1.29 \\
\hline 7 & 1250 & 543 & 478 & 1.14 \\
\hline 8 & 1600 & 567 & 531 & 1.07 \\
\hline 9 & 2000 & 605 & 580 & 1.04 \\
\hline 10 & 2500 & 649 & 632 & 1.03 \\
\hline 11 & 3150 & 697 & 685 & 1.02 \\
\hline 12 & 4000 & 747 & 739 & 1.01 \\
\hline
\end{tabular}

$$
\begin{aligned}
& \frac{\left(A_{11} A_{22}-A_{12}^{2}\right) c^{4}}{a^{2}}
\end{aligned}
$$

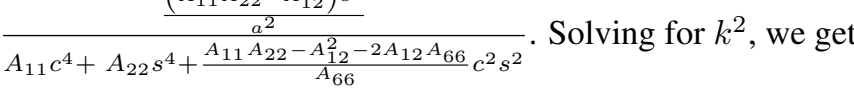

$$
\begin{aligned}
& k^{2}=\frac{\rho_{m} \omega_{m n}^{2}-f_{3}}{2 f_{1}}\left\{\frac{f_{2}}{N} \pm \sqrt{\left(\frac{f_{2}}{N}\right)^{2}+\frac{4 f_{1}}{\rho_{m} \omega_{m n}^{2}-f_{3}}}\right\}
\end{aligned}
$$

Average value of the wave number at a given frequency can be obtained as below

$$
\begin{aligned}
& k=\frac{2}{\pi} \int_{0}^{\frac{\pi}{2}} \\
& {\left[\frac{\rho_{m} \omega^{2}-f_{3}}{2 f_{1}}\left\{\frac{f_{2}}{N}+\sqrt{\left(\frac{f_{2}}{N}\right)^{2}+\frac{4 f_{1}}{\rho_{m} \omega^{2}-f_{3}}}\right\}\right]^{\frac{1}{2}} d \theta .}
\end{aligned}
$$

Wave number for a plate can be found by setting radius to infinity. This implies that the function $f_{3}=0$

$$
k_{\text {plate }}=\frac{2}{\pi} \int_{0}^{\frac{\pi}{2}}\left[\frac{\rho_{m} \omega^{2}}{2 f_{1}}\left\{\frac{f_{2}}{N}+\sqrt{\left(\frac{f_{2}}{N}\right)^{2}+\frac{4 f_{1}}{\rho_{m} \omega^{2}}}\right\}\right]^{\frac{1}{2}} d \theta .
$$

Wavenumbers are now computed for the composite thick cylinder and equivalent thick plate using Eqs. (46) and (47) respectively. The speed of the bending wave is then obtained from the computed wavenumber at a specific frequency. Results are summarized in Table 3.

The results show a similar behaviour as discussed in section 6.1. The critical frequency of the shell is about $450 \mathrm{~Hz}$, taking $343 \mathrm{~m} / \mathrm{s}$ as the speed of sound in air. It is about $600 \mathrm{~Hz}$ if calculated neglecting the curvature. The results show the influence of the curvature and the need for the expression derived. The critical frequencies estimated using the expressions derived in this work are $495 \mathrm{~Hz}$ and $600 \mathrm{~Hz}$ respectively for the cylindrical shell and plate approximation. The results are in agreement with the characteristics obtained using the expressions derived in this work

The speed of the bending waves in thin/thick composite shells and equivalent flat plates are summarized in Fig. 1. One can see that

1. Curvature increases the flexural wave speed. This effect is more pronounced at low frequencies. This influence is negligible at frequencies well above the ring frequencies. 


$$
\begin{aligned}
& \omega_{m n}^{2}=\frac{1}{\rho_{m}\left[1+\left(\frac{D_{11}}{N}\left(\frac{m \pi}{L}\right)^{2}+\frac{D_{22}}{N}\left(\frac{n}{a}\right)^{2}\right)\right]}\left\{D_{11}\left(\frac{m \pi}{L}\right)^{4}+2\left(D_{12}+2 D_{66}\right)\left(\frac{m \pi}{L}\right)^{2}\left(\frac{n}{a}\right)^{2}+\right. \\
& \left.D_{22}\left(\frac{n}{a}\right)^{4}+\frac{\frac{A_{11} A_{22}-A_{12}^{2}}{a}\left(\frac{m \pi}{L}\right)^{2} \times\left\{\frac{1}{a}\left(\frac{m \pi}{L}\right)^{2}+\frac{1}{a N}\left(D_{11}\left(\frac{m \pi}{L}\right)^{4}+D_{22}\left(\frac{m \pi}{L}\right)^{2}\left(\frac{n}{a}\right)^{2}\right)\right\}}{A_{11}\left(\frac{m \pi}{L}\right)^{4}+A_{22}\left(\frac{n}{a}\right)^{4}+\frac{A_{11} A_{22}-A_{12}^{2}-2 A_{12} A_{66}}{A_{66}}\left(\frac{m \pi}{L}\right)^{2}\left(\frac{n}{a}\right)^{2}}\right\} .
\end{aligned}
$$

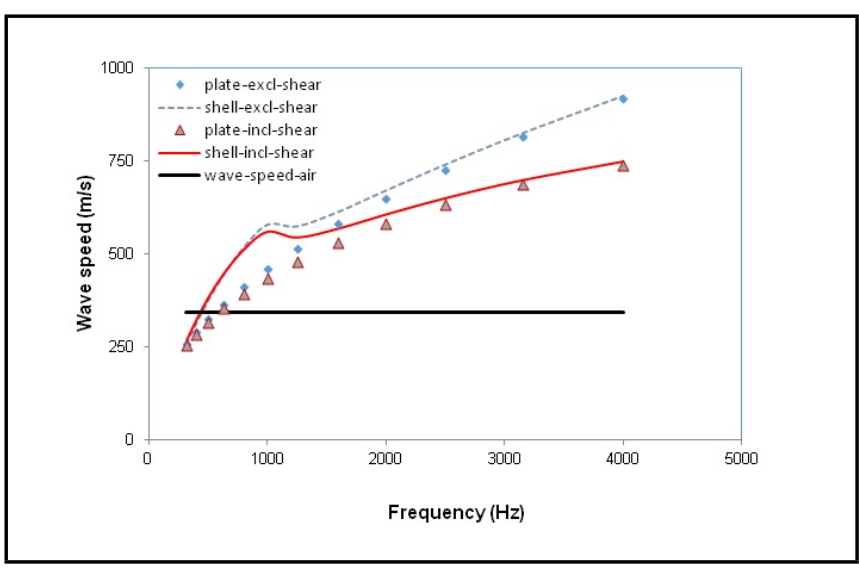

Figure 1. Speed of bending waves in composite cylinders and flat plates.

2. The speed of the bending wave decreases with transverse shear deformation.

\section{EXPERIMENTAL RESULTS}

An attempt is made here to determine the critical frequency experimentally. Influence of the critical frequency on the total loss factor is used in identifying the presence of the critical frequency.

At critical frequency, the sound radiation and hence the radiation loss factor is quite significant. Experimentally, determining the radiation loss factor needs much more instrumentation, such as measurement of sound pressure level etc. A simple method is to determine the total loss factor which is the sum of the radiation loss factor and the dissipation loss factor. The total loss factor will show a sudden increase near the critical frequency as the radiation loss factor is very high near the critical frequency and change in the dissipation loss factor with frequency is very less. This characteristic is used in determining the critical frequency. ${ }^{11}$ The total loss factor of the structure can be experimentally determined by using a shaker system for point excitation and measuring the acceleration responses at various locations and the input power. By equating the input power with the total power loss, the total loss factor can be determined.

The cylinder whose geometry/structural properties are given in section 5 is taken for the experiments. The cylinder is hung in a reverberation chamber and driven mechanically at three locations, one point at a time. Fig. 2 shows the test setup. The force and the drive point acceleration are measured during each excitation by means of the impedance head. During excitation at each drive point, the acceleration responses are measured at 9 randomly selected locations using piezo-electric accelerometers. The spatial average of these measured accelerations is computed for each drive point.

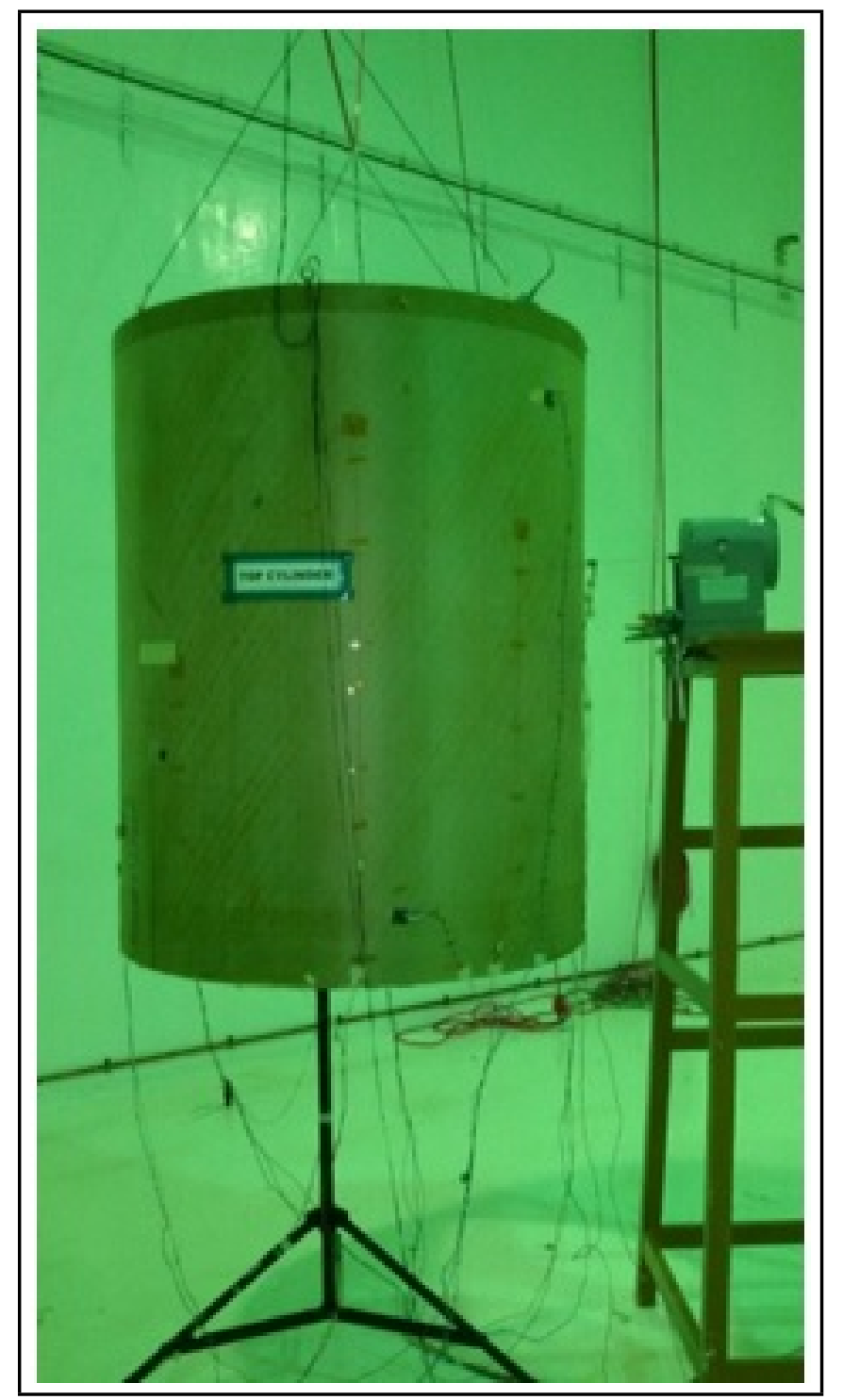

Figure 2. Test setup.

From the above measured results, the total loss factor is determined from the relation.

$$
\eta_{\text {total }}=\frac{F_{a}^{2}<\operatorname{Re}\left(Y_{a}\right)>\omega}{\rho_{m} A<a^{2}>_{x}}
$$

The measured forces and the measured admittance need correction as given below. ${ }^{11}$ From the measured force, the actual force is

$$
\overline{F_{a}^{2}}=\frac{\overline{F_{m}^{2}}}{\left[1-\omega M \operatorname{Img}\left(Y_{a}\right)\right]^{2}+\left[\omega M \operatorname{Re}\left(Y_{a}\right)\right]^{2}} .
$$




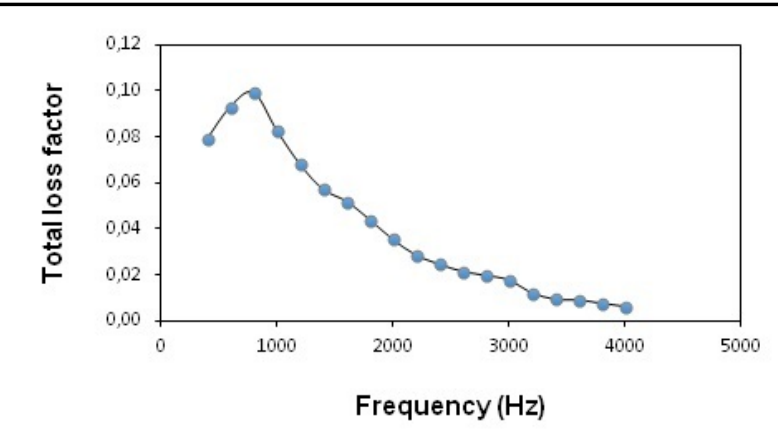

Figure 3. Total loss factor of the cylinder.

The real part of the actual admittance is

$$
\operatorname{Re}\left(Y_{a}\right)=\frac{\operatorname{Re}\left(Y_{m}\right)}{\left[1+\omega M \operatorname{Img}\left(Y_{m}\right)\right]^{2}+\left[\omega M \operatorname{Re}\left(Y_{m}\right)\right]^{2}} .
$$

The imaginary part of the actual admittance is

$$
\operatorname{Img}\left(Y_{a}\right)=\frac{\operatorname{Img}\left(Y_{m}\right)+\omega M\left[\operatorname{Img}\left(Y_{m}\right)^{2}+\operatorname{Re}\left(Y_{m}\right)^{2}\right]}{\left[1+\omega M \operatorname{Img}\left(Y_{m}\right)\right]^{2}+\left[\omega M \operatorname{Re}\left(Y_{m}\right)\right]^{2}} .
$$

To determine the total loss factor, the input power and the acceleration responses are averaged over the driving point locations. The total loss factor obtained is shown in Fig. 3.

One can see an increase in the loss factor around $550 \mathrm{~Hz}$. The theoretically estimated critical frequency of this cylinder is about $500 \mathrm{~Hz}$. It is to be noted that the critical frequency cannot be accurately determined through this method and the total loss factor depends on many factors other than the critical frequency. Also, the frequency bandwidth itself is $200 \mathrm{~Hz}$. Nevertheless, the test results show the presence of the critical frequency near $500 \mathrm{~Hz}$.

\section{CONCLUSIONS}

The critical frequency of the cylindrical shell determines its sound radiation characteristics. At present the critical frequencies of cylindrical shells are estimated using expressions for flat plates. However, this method is inaccurate because the speed of the bending wave is influenced by the curvature of the shell. In this work, an expression for determining the speed of the bending wave in a composite cylindrical shell is derived. It is seen that the curvature increases the speed of the bending wave meaning that the speed is higher than that of the bending wave in the equivalent plate. The orthotropic nature of the structure reduces the speed of the bending wave. Influence of transverse shear deformation is also incorporated in the above expression and it reduces the speed of the bending wave being influential at higher frequencies.

Expressions for estimating the critical frequency of composite cylindrical shells are then derived. The expressions are cast in a form such that they are related to critical frequencies of equivalent composite panels, i.e., cylindrical shell with infinite radius. The critical frequency gets reduced due to the curvature and the influence is significant for acoustically thick cylinders in which the critical frequency is lower than the ring frequency. The critical frequency of such cylinders cannot be determined by using the expression for the panels. The critical frequency of the shell can be approximated to the critical frequency of the panel, if the critical frequency is higher than the ring frequency. The critical frequency is increased due to the presence of transverse shear deformations, which are significant in honeycomb sandwich panels.

The expression derived for critical frequency is validated by comparing with the results of numerical simulations performed on a typical composite cylindrical shell and an agreement can be observed. The total loss factor of a typical composite cylinder is obtained through experiments that show the presence of critical frequency near the value estimated using the expression derived.

\section{REFERENCES}

1 Norton M.P. Fundamentals of Noise and Vibration Analysis for Engineers, Cambridge University Press, England, 1989.

2 Renji K., Nair P.S. and Narayanan S. Critical and coincidence frequencies of flat panels, Journal of Sound and Vibration, 205(1), 19-32, (1997). https://dx.doi.org/10.1006/jsvi.1997.0992

3 J.E.Manning andG. Maidanik. Radiation properties of Cylindrical Shells, Journal of the Acoustical society of America, 36, 1691-1698, (1964). https://dx.doi.org/10.1121/1.1919266

4 Szechenyi E. Modal densities and radiation efficiencies of unstiffened cylinders using statistical methods, Journal of Sound and Vibration, 19, 65-81, (1971). https://dx.doi.org/10.1016/0022-460X(71)90423-8

5 Ferguson N.S. and Clarkson B.L. The Modal density of Honeycomb shells, Transactions of the ASME, Journal of Vibration, Acoustics, Stress and Reliability in Design, 108, 399-404, (1986). https://dx.doi.org/10.1115/1.3269362

6 Ghinet S., Atalla N. and Osman H. Diffuse field transmission into infinite sandwich composite and laminate composite cylinders, Journal of Sound and Vibration, 289, 745778, (2006). https://dx.doi.org/10.1016/j.jsv.2005.02.028

7 Soedel W. Vibration of Shells and Plates, Marcel Dekker, New York, 1981.

8 Florence S.J.K and Renji K. Modal density of thin composite cylindrical shells, Journal of Sound and Vibration, 365, 157-171, (2016). https://dx.doi.org/10.1016/j.jsv.2015.11.030

9 Jones R.M. Mechanics of Composite materials, McGrawHill, New York, 1975.

10 Florence S.J.K, Renji K and Subramanian K. Modal density of honeycomb sandwich composite cylindrical shells considering transverse shear deformation, International Journal of Acoustics and Vibration, 23(1), 83-92, (2018).

11 Renji K. and Narayan S.S. Loss factors of honeycomb sandwich panels, Journal of Sound and Vibration, 250(4), 745761, (2002). https://dx.doi.org/10.1006/jsvi.2001.3602. 